Research Article

\title{
Exact Traveling Wave Solutions of the Gardner Equation by the Improved $\tan (\Theta(9))$-Expansion Method and the Wave Ansatz Method
}

\author{
Hatıra Günerhan (iD) ${ }^{1,2}$ \\ ${ }^{1}$ Young Researchers Club, Azad University bonab, Bonab, Iran \\ ${ }^{2}$ Department of Mathematics, Faculty of Education, Kafkas University, Kars, Turkey \\ Correspondence should be addressed to Hatıra Günerhan; hatira.gunerhan@kafkas.edu.tr
}

Received 4 August 2020; Revised 13 September 2020; Accepted 18 September 2020; Published 9 October 2020

Academic Editor: S. A. Edalatpanah

Copyright (c) 2020 Hatıra Günerhan. This is an open access article distributed under the Creative Commons Attribution License, which permits unrestricted use, distribution, and reproduction in any medium, provided the original work is properly cited.

Nonlinear partial differential equations (NLPDEs) are an inevitable mathematical tool to explore a large variety of engineering and physical phenomena. Due to this importance, many mathematical approaches have been established to seek their traveling wave solutions. In this study, the researchers examine the Gardner equation via two well-known analytical approaches, namely, the improved $\tan (\Theta(\vartheta))$-expansion method and the wave ansatz method. We derive the exact bright, dark, singular, and $W$-shaped soliton solutions of the Gardner equation. One can see that the methods are relatively easy and efficient to use. To better understand the characteristics of the theoretical results, several numerical simulations are carried out.

\section{Introduction}

The Gardner equation is given as [1-3]

$$
u_{t}+2 \alpha u u_{x}+3 \beta u^{2} u_{x}+\gamma u_{x x x}=0,
$$

where $\alpha, \beta$, and $(\gamma>0)$ are constant values. If the coefficient $\beta>0$, equation (1) admits two families of solitons and oscillating wave packets (called breathers), whereas if $\beta<0$, only one category of solitons exists [4].

Equation (1) is also called the combined KdV-mKdV equation. In recent years, partial differential equations have become one of the most widely used fields of mathematics in various branches of science and engineering [5-12]. In this paper, the Gardner equation is examined by using the improved $\tan (\Theta(\vartheta))$-expansion method and the wave ansatz method. Recently, the improved $\tan (\Theta(\vartheta))$-expansion method (ITEM) [13-16] and the wave ansatz method [17-21] have been exploited to integrate a variety of nonlinear partial differential evolution equations (NLPDEs). In the past, several years ago, various methods have been proposed to obtain the solitary solution of this equation. In $[22,23]$, some kind of solutions of equation (1) were obtained. The Gardner equation (1) for $\alpha>0, \beta<0$, and $\gamma=0$ has been studied in [24]. In [25], the authors have studied the attitudes of some solitary solitons for this equation. Many powerful analytic solution methods for solving nonlinear equation (1) have appeared in the open literature, such as the Hirota bilinear method [26], mapping method [25], similarity transformation method [27], generalized exponential rational function method, Jacobi elliptical solution finder method [28], fractional homotopy perturbation transform method [29], Coffey's series expansion method [30], a unified method including solitary wave solutions, triangular periodic solutions, and Jacobi periodic wave solutions, as well as rational solutions [23], Wadati's inverse scattering transform and Hirota methods [31, 32], consistent Riccati expansion (CRE) [33], planar dynamical systems approach method [34], Kudryashov method [35], Lie symmetry group method [36], ill-posedness results [37], classification of single traveling wave solutions [38], spectral collocation method [5], the Gardner equation with time-dependent coefficients and forcing term, have been investigated in $[39,40]$. For more methods, we refer the readers to [22-40] and the references therein. 
This paper consists of several sections. In Section 2, a brief description of the improved $\tan (\Theta(\vartheta))$-expansion method is reviewed. With the aid of this method, we will retrieve several sets of solutions for the Gardner equation in Section 3. In Section 4, the method of wave ansatz method is considered and the corresponding solutions in terms of bright, dark, singular, and $W$-shaped soliton solutions. Furthermore, some 3D profiles of acquired solutions are also depicted in this section. However, to the best of the authors' knowledge, these two approaches have not been applied for equation (1) in previous studies. Finally, Section 5 concludes the paper.

\section{The Improved $\tan (\Theta(9))$-Expansion Method}

In this section, the main algorithm of the improved $\tan (\Theta(\vartheta))$-expansion method (ITEM) is explained as follows:

Step 1: using a new definition of wave variable $(\vartheta=\mu x-\theta t)$, a general partial differential equation (PDE) such as

$$
\mathcal{N}\left(u, u_{x}, u_{t}, u_{x x}, \cdots\right)=0
$$

is transformed into an ordinary differential equation (ODE)

$$
\mathcal{N}\left(u, u^{\prime},-\mu u^{\prime}, u^{\prime \prime}, \mu^{2} u^{\prime \prime}, \cdots\right)=0 .
$$

Step 2: suppose that

$$
\begin{aligned}
u(\vartheta)= & S(\Theta)=\sum_{k=0}^{m} A_{k}\left[p+\tan \left(\frac{\Theta(\vartheta)}{2}\right)\right]^{k} \\
& +\sum_{k=1}^{m} B_{k}\left[p+\tan \left(\frac{\Theta(\vartheta)}{2}\right)\right]^{-k}
\end{aligned}
$$

could be constructed as a solution of equation (2), where $\left(A_{k}(0 \leq k \leq m)\right)$ and $\left(B_{k}(1 \leq k \leq m)\right)$ with $A_{m} \neq 0, B_{m} \neq 0$ are unknown parameters, so that $\Theta=\Theta(\vartheta)$ satisfies

$$
\Theta^{\prime}(\vartheta)=a \sin (\vartheta)+b \cos (\vartheta)+c .
$$

Taking (5) into account, some solutions are as follows:

Category 1: while $\left(a^{2}+b^{2}-c^{2}<0\right)$ and $b-c \neq 0$, then

$$
\begin{aligned}
\Theta(\vartheta)= & -2 \tan ^{-1}\left[-\frac{a}{b-c}+\frac{\sqrt{c^{2}-a^{2}-b^{2}}}{b-c}\right. \\
& \left.\cdot \tan \left(\frac{\sqrt{c^{2}-a^{2}-b^{2}}}{2}(\vartheta+C)\right)\right] .
\end{aligned}
$$

Category 2: while $a^{2}+b^{2}-c^{2}>0$ and $b-c \neq 0$, then

$$
\begin{aligned}
\Theta(\vartheta)= & -2 \tan ^{-1}\left[-\frac{a}{b-c}-\frac{\sqrt{b^{2}+a^{2}-c^{2}}}{b-c}\right. \\
& \left.\cdot \tanh \left(\frac{\sqrt{b^{2}+a^{2}-c^{2}}}{2}(\vartheta+C)\right)\right] .
\end{aligned}
$$

Category 3: while $\left(a^{2}+b^{2}-c^{2}>0\right), b \neq 0$, and $c=0$, then

$\Theta(\vartheta)=2 \tan ^{-1}\left[\frac{a}{b}+\frac{\sqrt{b^{2}+a^{2}}}{b} \tanh \left(\frac{\sqrt{b^{2}+a^{2}}}{2}(\vartheta+C)\right)\right]$.

Category 4: while $\left(a^{2}+b^{2}-c^{2}<0\right), c \neq 0$, and $b=0$, then

$\Theta(\vartheta)=2 \tan ^{-1}\left[-\frac{a}{c}+\frac{\sqrt{c^{2}-a^{2}}}{c} \tan \left(\frac{\sqrt{c^{2}-a^{2}}}{2}(\vartheta+C)\right)\right]$.

Category 5: while $\left(a^{2}+b^{2}-c^{2}>0, b-c \neq 0\right)$ and $a=0$, then

$$
\Theta(\vartheta)=2 \tan ^{-1}\left[\sqrt{\frac{b+c}{b-c}} \tanh \left(\frac{\sqrt{b^{2}-c^{2}}}{2}(\vartheta+C)\right)\right] .
$$

Category 6: while $a=0$ and $c=0$, it resulted that

$$
\Theta(\vartheta)=\tan ^{-1}\left[\frac{e^{2 b(\vartheta+C)}-1}{e^{2 b(\vartheta+C)}+1}, \frac{2 e^{b(\vartheta+C)}}{e^{2 b(\vartheta+C)}+1}\right] .
$$

Category 7: while $b=0$ and $c=0$, it resulted that

$$
\Theta(\vartheta)=\tan ^{-1}\left[\frac{2 e^{a(9+C)}}{e^{2 a(\vartheta+C)}+1}, \frac{e^{2 a(\vartheta+C)}-1}{e^{2 a(\vartheta+C)}+1}\right] .
$$

Category 8: while $a^{2}+b^{2}=c^{2}$, it resulted that

$$
\Theta(\vartheta)=-2 \tan ^{-1}\left[\frac{(b+c)(a(\vartheta+C)+2)}{a^{2}(\vartheta+C)}\right] .
$$

Category 9: while $a=b=c=\kappa$, it resulted that

$$
\Theta(\vartheta)=2 \tan ^{-1}\left[e^{\kappa(\vartheta+C)}-1\right] .
$$

Category 10: while $a=c=\kappa$ and $b=-\kappa$, it resulted that

$$
\Theta(\vartheta)=-2 \tan ^{-1}\left[\frac{e^{\kappa(9+C)}}{-1+e^{k \delta(9+C)}}\right] .
$$

Category 11: while $c=a$, it resulted that

$$
\Theta(\vartheta)=-2 \tan ^{-1}\left[\frac{(a+b) e^{b(\vartheta+C)}-1}{(a-b) e^{b(\vartheta+C)}-1}\right] .
$$


Category 12: while $a=c$, it resulted that

$$
\Theta(\vartheta)=2 \tan ^{-1}\left[\frac{(b+c) e^{b(9+C)}+1}{(b-c) e^{b(9+C)}-1}\right] .
$$

Category 13: while $c=-a$, it resulted that

$$
\Theta(\vartheta)=2 \tan ^{-1}\left[\frac{e^{b(\vartheta+C)}+b-a}{e^{b(\vartheta+C)}-b-a}\right] .
$$

Category 14: while $b=-c$, it resulted that

$$
\Theta(\vartheta)=-2 \tan ^{-1}\left[\frac{a e^{a(\vartheta+C)}}{c e^{a(\vartheta+C)}-1}\right] .
$$

Category 15: while $b=0$ and $a=c$, it resulted that

$$
\Theta(\vartheta)=-2 \tan ^{-1}\left[\frac{c(\vartheta+C)+2}{c(\vartheta+C)}\right] .
$$

Category 16: while $a=0$ and $b=c$, it resulted that

$$
\Theta(\vartheta)=2 \tan ^{-1}[c(\vartheta+C)] .
$$

Category 17: while $a=0$ and $b=-c$, it resulted that

$$
\Theta(\vartheta)=-2 \tan ^{-1}\left[\frac{1}{c(\vartheta+C)}\right] \text {. }
$$

Category 18: while $a=0$ and $b=0$, it resulted that

$$
\Theta(\vartheta)=c \vartheta+C \text {. }
$$

Category 19: while $b=c$, it resulted that

$$
\Theta(\vartheta)=2 \tan ^{-1}\left[\frac{e^{a(9+C)}-c}{a}\right],
$$

where $\left(A_{k}, B_{k}(k=1,2, \cdots, m), a, b\right)$ and $c$ are the unknown parameters that need to be calculated. To determine the natural number $m$, one can use the homogeneous balance rule.

Step 3: inserting the formal scheme of (4) into equation (3), and then setting each coefficient of $\tan ((\Theta(\vartheta)) / 2)^{k}, \cot ((\Theta(\vartheta)) / 2)^{k},(k=0,1,2, \cdots)$ to zero, we will arrive to a set of nonlinear equations for $\left(A_{0}, A_{k}, B_{k}(k=1,2, \cdots, m), a, b, c, \mu, \theta\right)$ and $p$.

Step 4: solving the algebraic equations in Step 3, it resulted that substituting $\left(A_{0}, A_{1}, B_{1}, \cdots, A_{m}\right.$, $\left.B_{m}, \mu, \theta, p\right)$ in (4).

\section{Applications of the Gardner Equation via ITEM}

In this section, we will examine ITEM for equation (1). To find the traveling solutions for equation (1), we define the wave transformation as $u=U(\vartheta)$, where $\vartheta=\mu x-\theta t, \mu \neq 0$, and $\theta \neq 0$ to be determined later. Taking $u=u(\vartheta)$ into account allows us to rewrite equation (1) as the following ordinary differential equation:

$$
-\theta u^{\prime}+2 \alpha \mu u u^{\prime}+3 \beta \mu u^{2} u^{\prime}+\gamma \mu^{3} u^{\prime \prime \prime}=0 .
$$

Integrating (25) once with respect to $\vartheta$ and neglecting the resulted integration constants, we obtain

$$
-\theta u+\alpha \mu u^{2}+\beta \mu u^{3}+\gamma \mu^{3} u^{\prime \prime}=0 .
$$

Now, we apply the ITEM to obtain traveling wave solutions of the Gardner equation (1). According to this method, the solution of equation (26) can be written in the form of equation (4).

Balancing the $u^{\prime \prime}$ and $u^{3}$ in (26), by using homogeneous, one has

$$
3 m=m+2 \Longrightarrow m=1 \text {. }
$$

Taking $p=0$ in (27), the solution structure is formulated as

$$
u(\vartheta)=A_{0}+A_{1}\left[\tan \left(\frac{\Theta(\vartheta)}{2}\right)\right]+B_{1}\left[\cot \left(\frac{\Theta(\vartheta)}{2}\right)\right] .
$$

Substituting equation (28) into equation (26) and following the necessary steps of ITEM, we have the following sets of coefficients for the nontrivial solutions of (1) as follows:

Set 1:

$$
\begin{aligned}
\mu & =-\frac{\sqrt{2} \alpha}{3 \sqrt{-\sigma \beta} \sqrt{a^{2}+b^{2}-c^{2}}}, \\
\theta & =\frac{2 \alpha^{3} \sqrt{2}}{27 \beta \sqrt{-\sigma \beta} \sqrt{a^{2}+b^{2}-c^{2}}}, \\
A_{0} & =-\frac{\alpha}{3 \beta}\left(1+a \sqrt{b^{2}+a^{2}-c^{2}}\right), \\
A_{1} & =\frac{\alpha}{3 \beta} \frac{b-c}{\sqrt{b^{2}+a^{2}-c^{2}}}, \\
B_{1} & =0, \\
u(\vartheta) & =A_{0}+A_{1}\left[\tan \left(\frac{\Theta(\vartheta)}{2}\right)\right],
\end{aligned}
$$

where $a, b$, and $c$ are optional constants, and

$\vartheta=-\frac{\sqrt{2} \alpha}{3 \sqrt{-\beta \gamma} \sqrt{b^{2}+a^{2}-c^{2}}} x-\frac{2 \sqrt{2} \alpha^{3}}{27 \beta \sqrt{-\beta \gamma} \sqrt{b^{2}+a^{2}-c^{2}}} t$,

provided that $\beta<0$.

Setting these values in categories $2,6,10$, and 14 of Section 2, respectively, we acquire the following solutions: 
$u_{1}(\vartheta)=\frac{\alpha}{3 \beta}\left(\tanh \left(\frac{\sqrt{a^{2}+b^{2}-c^{2}}}{2} \vartheta\right)-1\right)$

where $a^{2}+b^{2}-c^{2}>0$ and $\vartheta$ is given by (32).

$u_{2}(\vartheta)=-\frac{\alpha}{3 \beta}\left[1+\tan \left(12 \tan ^{-1}\left(\frac{e^{2 b 9}-1}{e^{2 b 9}+1}, \frac{2 e^{b 9}}{e^{2 b 9}+1}\right)\right)\right]$,

where $\quad \vartheta=-(\sqrt{2} \alpha /(3 b \sqrt{-\beta \gamma})) x-\left(\left(2 \sqrt{2} \alpha^{3}\right) /\right.$ $(27 b \beta \sqrt{-\beta \gamma})) t$.

$$
u_{3}(9)=-\frac{2 \alpha}{3 \beta} \frac{c e^{a \vartheta}}{c e^{a 9}-1},
$$

where $\quad \vartheta=-(\sqrt{2} \alpha /(3 b \sqrt{-\beta \gamma})) x-\left(\left(2 \sqrt{2} \alpha^{3}\right) /\right.$ $(27 b \beta \sqrt{-\beta \gamma})) t$.

Set 2:

$$
\begin{aligned}
\mu & =-\frac{\sqrt{2} \alpha}{3 \sqrt{-\sigma \beta} \sqrt{a^{2}+b^{2}-c^{2}}}, \\
\theta & =\frac{2 \alpha^{3} \sqrt{2}}{27 \beta \sqrt{-\sigma \beta} \sqrt{a^{2}+b^{2}-c^{2}}}, \\
A_{0} & =-\frac{\alpha}{3 \beta}\left(1+a \sqrt{b^{2}+a^{2}-c^{2}}\right), \\
A_{1} & =0, \\
B_{1} & =-\frac{\alpha}{3 \beta} \frac{b+c}{\sqrt{b^{2}+a^{2}-c^{2}}}, \\
u(9) & =A_{0}+B_{1}\left[\cot \left(\frac{\Theta(\vartheta)}{2}\right)\right],
\end{aligned}
$$

where $a, b$, and $c$ are optional constants, and

$\vartheta=-\frac{\sqrt{2} \alpha}{3 \sqrt{-\beta \gamma} \sqrt{b^{2}+a^{2}-c^{2}}} x-\frac{2 \sqrt{2} \alpha^{3}}{27 \beta \sqrt{-\beta \gamma} \sqrt{b^{2}+a^{2}-c^{2}}} t$,

provided that $\beta<0$.

Setting these values in categories 3,5 , and 6 of Section 2 , respectively, we obtain

$u_{4}(\vartheta)=-\frac{\alpha}{3 \beta} \frac{\left(1+\tanh \left(\left(\sqrt{a^{2}+b^{2}} / 2\right) \vartheta\right)\right)\left(\sqrt{a^{2}+b^{2}}+a\right)}{a+\sqrt{a^{2}+b^{2}} \tanh \left(\left(\sqrt{a^{2}+b^{2}} / 2\right) \vartheta\right)}$,

where $9=-\left(\sqrt{2} \alpha /\left(3 \sqrt{-\beta \gamma} \sqrt{a^{2}+b^{2}}\right)\right) x-\left(\left(2 \sqrt{2} \alpha^{3}\right) /\right.$ $\left.\left(27 \beta \sqrt{-\beta \gamma} \sqrt{a^{2}+b^{2}}\right)\right) t$.

$$
u_{5}(\vartheta)=-\frac{\alpha}{3 \beta} \frac{\tanh \left(\left(\left(\sqrt{b^{2}-c^{2}}\right) / 2\right) \vartheta\right)+1}{\tanh \left(\left(\left(\sqrt{b^{2}-c^{2}}\right) / 2\right) \vartheta\right)}
$$

where $b^{2}-c^{2}>0$ and $\vartheta=-\left(\sqrt{2} \alpha /\left(3 \sqrt{-\beta \gamma} \sqrt{b^{2}-c^{2}}\right)\right)$ $x-\left(2 \sqrt{2} \alpha^{3} /\left(27 \beta \sqrt{-\beta \gamma} \sqrt{b^{2}-c^{2}}\right)\right) t$.

$$
u_{6}(9)=-\frac{\alpha}{3 \beta} \frac{\tan \left(12 \tan ^{-1}\left(\left(e^{2 b 9}-1 / e^{2 b 9}+1\right), 2\left(e^{b \vartheta} / e^{2 b 9}+1\right)\right)\right)-1}{\tan \left(12 \tan ^{-1}\left(\left(e^{2 b 9}-1 / e^{2 b 9}+1\right), 2\left(e^{b 9} / e^{2 b 9}+1\right)\right)\right)},
$$

where $9=-(\sqrt{2} \alpha / 3 b \sqrt{-\beta \gamma}) x-\left(2 \sqrt{2} \alpha^{3} / 27 b \beta \sqrt{-\beta \gamma}\right) t$. Set 3:

$$
\begin{aligned}
& a=c=0, \\
& \mu=\frac{\sqrt{2} \alpha}{6 b \sqrt{-\gamma \beta},} \\
& \theta=-\frac{\sqrt{2} \alpha^{3}}{27 b \beta \sqrt{-\gamma \beta},} \\
& A_{0}=-\frac{\alpha}{3 \beta}, \\
& A_{1}=-\frac{\alpha}{6 \beta}, \\
& B_{1}=-\frac{\alpha}{6 \beta}, \\
& u(\vartheta)=A_{0}+A_{1}\left[\tan \left(\frac{\Theta(9)}{2}\right)\right]+B_{1}\left[\cot \left(\frac{\Theta(9)}{2}\right)\right],
\end{aligned}
$$

where $b$ is an optional and $\beta<0$ must be held.

Setting these values in categories 1,6 , and 13 of Section 2 , respectively, we obtain

$$
\begin{aligned}
& u_{7}=-\frac{\alpha}{6 \beta} \frac{(\tanh (b / 29)+1)^{2}}{\tanh (b 29)}, \\
& u_{8}=-\frac{\alpha}{6 \beta} \frac{\left(\tan \left(12 \tan ^{-1}\left(\left(e^{2 b 9}-1 / e^{2 b 9}+1\right), 2\left(e^{b 9} / e^{2 b 9}+1\right)\right)\right)+1\right)^{2}}{\tan \left(12 \tan ^{-1}\left(\left(e^{2 b 9}-1 / e^{2 b 9}+1\right), 2\left(e^{b 9} / e^{2 b 9}+1\right)\right)\right)}, \\
& u_{9}=-\frac{2 \alpha}{3 \beta} \frac{e^{2 b 9}}{e^{2 b 9}-b^{2}},
\end{aligned}
$$

where

$$
\vartheta=\frac{\sqrt{2} \alpha}{6 b \sqrt{-\gamma \beta}}+\frac{\sqrt{2} \alpha^{3}}{27 b \beta \sqrt{-\gamma \beta}} t .
$$


It is worth to note that one can find some more new exact solitary solutions from solutions (31), (38), and (45).

\section{Applications of the Wave Ansatz Method}

In what follows, and based on the wave ansatz method, several types of soliton wave solutions for the Gardner equation (1) are presented which is based on the wave ansatz method (see the previous study [24]).

4.1. Bright Soliton. To retrieve bright optical solutions of the Gardner equation, we use the following scheme [41]:

$$
u(x, t)=\frac{A}{(D+\cosh \tau)^{n}},
$$

where

$$
\tau=B(x-v t)
$$

where $A, B$, and $v$ are disposal parameters.

Putting these values of (48) into (1) and some calculations, one obtains

$$
\begin{aligned}
& -\frac{3 \beta A^{3} n B \sinh \tau}{(D+\cosh \tau)^{3 n+1}}-\frac{2 \alpha A^{2} n B \sinh \tau}{(D+\cosh \tau)^{2 n+1}} \\
& -\frac{A B^{3} \gamma n(n+2)(n+1)\left(D^{2}-1\right) \sinh \tau}{(D+\cosh \tau)^{n+3}} \\
& +\frac{A B^{3} D \gamma n(n+1)(2 n+1) \sinh \tau}{(D+\cosh \tau)^{n+2}} \\
& -\frac{A B n\left(B^{2} \gamma n^{2}-v\right) \sinh \tau}{(D+\cosh \tau)^{n+1}}=0 .
\end{aligned}
$$
yields

From (50), equating the exponents $3 n+1$ and $n+3$

$$
n=1 \text {. }
$$

So, solving equation (50) turns to the following equation:

$$
\begin{aligned}
& -\frac{A B\left(B^{2} \gamma-\nu\right)}{(D+\cosh \tau)^{2}}-\frac{2 A B\left(-3 B^{2} D \gamma+A \alpha\right)}{(D+\cosh \tau)^{3}} \\
& -\frac{3 A B\left(A^{2} \beta+2 B^{2} \gamma D^{2}-2 B^{2} \gamma\right)}{(D+\cosh \tau)^{4}}=0 .
\end{aligned}
$$

Due to the fact that the functions $\left(1 /(D+\cosh \tau)^{j}\right)$ for $j=2,3$, and 4 are linearly independent, equation (52) will introduce a system of equations for the unknown parameters. Solving this system, one gets

$$
\begin{aligned}
& \nu=\gamma B^{2} \\
& A=\frac{3 \gamma B^{2} D}{\alpha} \\
& D=\frac{2 \alpha}{\sqrt{18 \beta \gamma B^{2}+4 \alpha^{2}}} .
\end{aligned}
$$

Putting (55) into (54) yields

$$
A=\frac{6 \gamma B^{2}}{\sqrt{18 \beta \gamma B^{2}+4 \alpha^{2}}} \text {. }
$$

Thus, for an arbitrary constant $B$, the 1 -soliton solution of (1) is given by

$$
u(x, t)=\frac{6 \gamma B^{2}}{2 \alpha+\sqrt{18 \beta \gamma B^{2}+4 \alpha^{2}} \cosh \left(B x-\gamma B^{3} t\right)},
$$

provided

$$
9 B^{2} \beta \gamma+2 \alpha^{2}>0 \text {. }
$$

4.2. Dark Soliton. To retrieve dark solutions of the equation, we use the structure [41]

$$
u(x, t)=(A+B \tanh \tau)^{n},
$$

where

$$
\tau=\mu(x-v t),
$$

where $A, B, \mu$, and $\nu$ are unknown parameters.

Inserting (59) into (1) gives 


$$
\begin{aligned}
& -\left[\frac{3 \beta n \mu}{B}\right](A+B \tanh \tau)^{3 n+1}+\left[\frac{6 \beta n \mu A}{B}\right](A+B \tanh \tau)^{3 n}-\left[\frac{3 \beta \mu n\left(A^{2}-B^{2}\right)}{B}\right] \\
& \cdot(A+B \tanh \tau)^{3 n-1}-\left[\frac{2 \alpha n \mu}{B}\right](A+B \tanh \tau)^{2 n+1} \\
& +\left[\frac{4 \alpha n \mu A}{B}\right](A+B \tanh \tau)^{2 n}-\left[\frac{2 \alpha \mu n\left(A^{2}-B^{2}\right)}{B}\right](A+B \tanh \tau)^{2 n-1}-\left[\frac{\gamma n \mu^{3}(n+2)(n+1)}{B^{3}}\right](A+B \tanh \tau)^{n+3} \\
& +\left[\frac{6 \gamma n \mu^{3} A(n+1)^{2}}{B^{3}}\right](A+B \tanh \tau)^{n+2}-\left[\frac{\mu n\left(3 \gamma \mu^{2} n\left(5 A^{2}-B^{2}\right)(n+1)+2 \gamma \mu^{2}\left(3 A^{2}-B^{2}\right)-B^{2} \nu\right)}{B^{3}}\right](A+B \tanh \tau)^{n+1} \\
& +\left[\frac{2 A \mu n\left(2 \gamma \mu^{2} n^{2}\left(5 A^{2}-3 B^{2}\right)+2 \gamma \mu^{2}\left(A^{2}-B^{2}\right)-B^{2} \nu\right)}{B^{3}}\right] \\
& \cdot(A+B \tanh \tau)^{n}-\left[\frac{\mu n\left(A^{2}-B^{2}\right)\left(3 \gamma \mu^{2} n\left(5 A^{2}-B^{2}\right)(n-1)+2 \gamma \mu^{2}\left(3 A^{2}-B^{2}\right)-B^{2} \nu\right)}{B^{3}}\right](A+B \tanh \tau)^{n-1} \\
& +\left[\frac{6 \gamma n \mu^{3} A(n-1)^{2}\left(A^{2}-B^{2}\right)^{2}}{B^{3}}\right](A+B \tanh \tau)^{n-2}-\left[\frac{\gamma n \mu^{3}(n-1)(n-2)\left(A^{2}-B^{2}\right)^{3}}{B^{3}}\right](A+B \tanh \tau)^{n-3}=0 \text {. }
\end{aligned}
$$

After some algebra, we conclude that

$$
\begin{aligned}
& \mu=\frac{\sqrt{-2 \gamma \beta}}{\gamma} B, \\
& \nu=\frac{3 B^{2} \beta^{2}-\alpha^{2}}{3 \beta}, \\
& A=-\frac{\alpha}{3 \beta} .
\end{aligned}
$$

The dark soliton solution of equation (1) is obtained as

$$
u(x, t)=(A+B \tanh (\mu(x-v t)))^{n},
$$

to exist, from (62), the following restriction is obtained

$$
\beta<0 \text {. }
$$

4.3. Singular Soliton. To extract the singular solitons of the Gardner equation (1), the following structure is examined by [41]

$$
u(x, t)=\frac{A}{(D+\sinh \tau)^{n}},
$$

with $\tau$ is defined by (49).

Substituting (67) into (1), we obtain

$$
\begin{aligned}
& -\frac{3 \beta A^{3} n B \cosh \tau}{(D+\sinh \tau)^{3 n+1}}-\frac{2 \alpha A^{2} n B \cosh \tau}{(D+\sinh \tau)^{2 n+1}} \\
& -\frac{A B^{3} \gamma n(n+2)(n+1)\left(D^{2}+1\right) \cosh \tau}{(D+\sinh \tau)^{n+3}} \\
& +\frac{A B^{3} D \gamma n(n+1)(2 n+1) \cosh \tau}{(D+\sinh \tau)^{n+2}} \\
& -\frac{A B n\left(B^{2} \gamma n^{2}-v\right) \cosh \tau}{(D+\sinh \tau)^{n+1}}=0 .
\end{aligned}
$$

Considering the balancing principle indicates (51), vanishing all the coefficients of $\left(\cosh \tau /[D+\sinh \tau]^{j}\right)$ for $j=$ 2,3 , and 4 to zero in (68), one gets

$$
\nu=B^{2} \gamma
$$

$$
\begin{aligned}
& A=\frac{6 B^{2} \gamma}{\sqrt{-18 B^{2} \beta \gamma-4 \alpha^{2}}}, \\
& D=\frac{2 \alpha}{\sqrt{-18 B^{2} \beta \gamma-4 \alpha^{2}}} .
\end{aligned}
$$

From (70) and (71), one concludes that if

$$
9 B^{2} \beta \gamma+2 \alpha^{2}<0
$$

holds, the soliton solution 


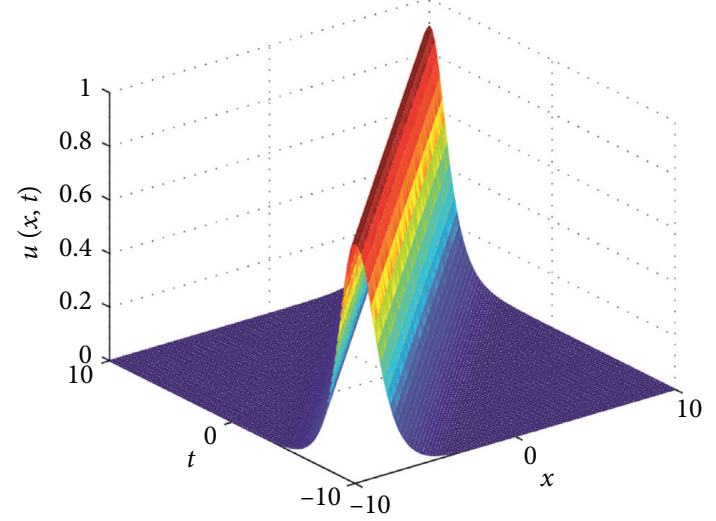

Figure 1: The $3 \mathrm{~d}$ surface of equation (57) for the values $\alpha=\beta=$ $\gamma=1$ and $B=1$.

$$
u(x, t)=\frac{A}{D+\sinh \left(B x-B^{3} \gamma t\right)}
$$

is achieved as a singular solution for the Gardner equation (1). In this solution, $A$ is given by (70), $D$ is shown in (71), and $B$ is an optional constant chosen in such a way that (72) holds.

4.4. W-Shaped Soliton. Now, we explore some exact solutions of the Gardner equation in the form of [41]

$$
u(x, t)=A+D \operatorname{sech}(\tau)
$$

where $\tau$ is the same as (49).

Substituting (74) into (1), we, respectively, obtain

$$
\begin{aligned}
& -B D \operatorname{sech}(\tau) \tanh (\tau)\left[\left(-6 B^{2} \gamma+3 D^{2} \beta\right) \operatorname{sech}^{2}(\tau)\right. \\
& \left.+(6 A D \beta+2 D \alpha) \operatorname{sech}(\tau)+3 A^{2} \beta+B^{2} \gamma+2 A \alpha-\nu\right]=0 .
\end{aligned}
$$

Now, equation (75) holds whenever we have

$$
\begin{aligned}
& \nu=\frac{3 B^{2} \beta \gamma-\alpha^{2}}{3 \beta}, \\
& A=-\frac{\alpha}{3 \beta}, \\
& D= \pm B \sqrt{\frac{2 \gamma}{\beta}},
\end{aligned}
$$

which will be valid for

$$
\beta<0 \text {. }
$$

Consequently, the solution (74) with sign " + " in equation (78) is obtained as

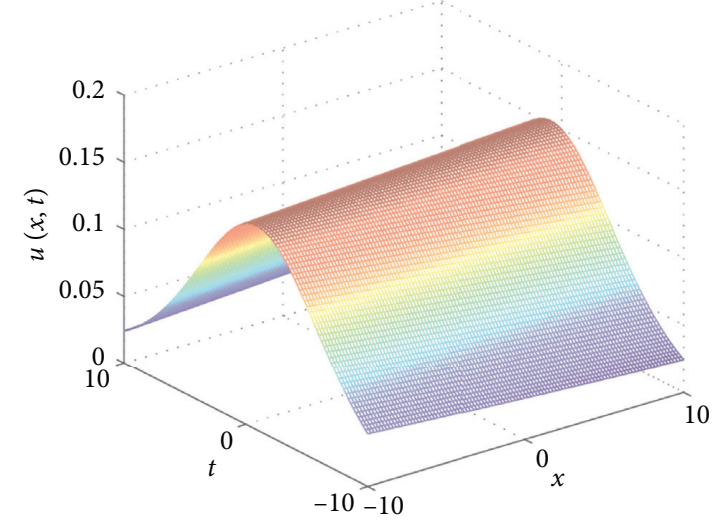

FIgURE 2: The 3d surface of equation (57) for the values $\alpha=\gamma=1, \beta=-1$, and $B=0.3$.

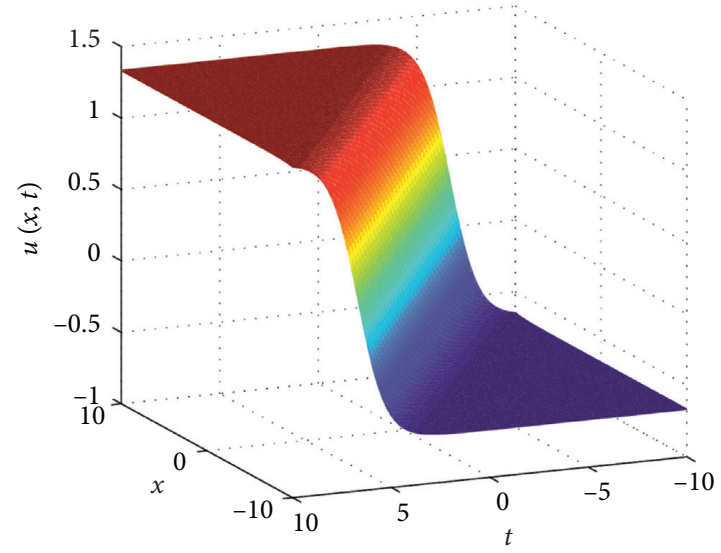

Figure 3: The 3d surface of equation (65) for the values $\alpha=\gamma=1, \beta=-1$, and $B=1$.

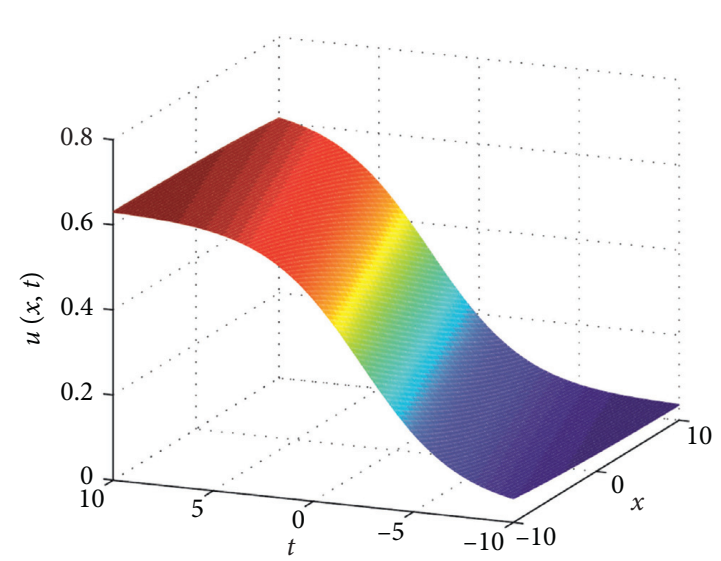

FIgURE 4: The 3d surface of equation (65) for the values $\alpha=\gamma=1, \beta=-1$, and $B=0.3$.

$$
u(x, t)=-\frac{\alpha}{3 \beta}+B \sqrt{\frac{2 \gamma}{\beta}} \operatorname{sech}\left(B x-\frac{3 B^{3} \beta \gamma-B \alpha^{2}}{3 \beta} t\right)
$$

Moreover, for bright soliton pulse and with sign “-” in equation (78), we obtain 


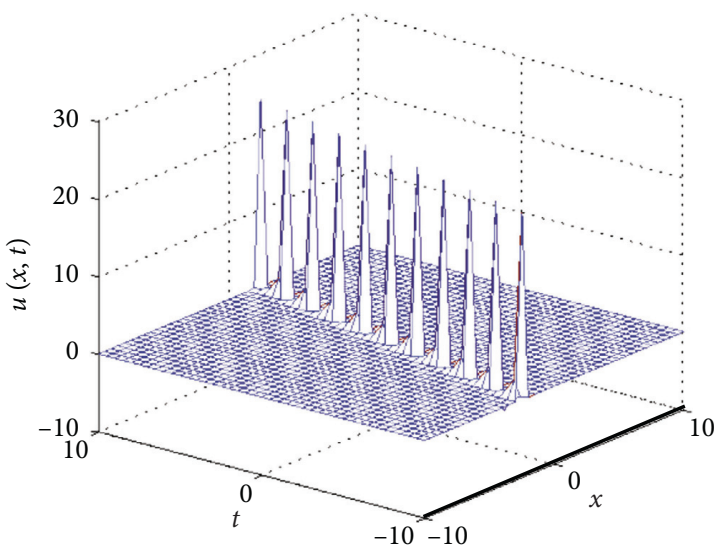

FIgURE 5: The 3d surface of equation (73) for the values $\alpha=0.5, \beta=-1, \gamma=1$, and $B=2$.

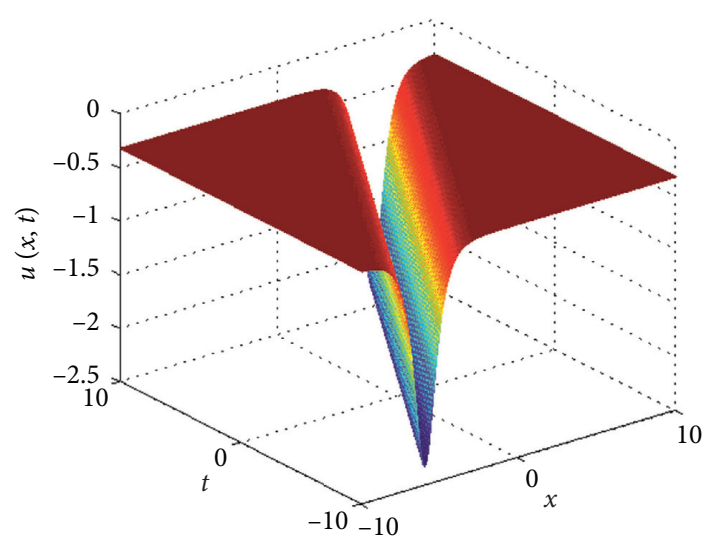

Figure 6: The 3d surface of equation (80) for the values $\alpha=\beta=1, \gamma=2$, and $B=1$.

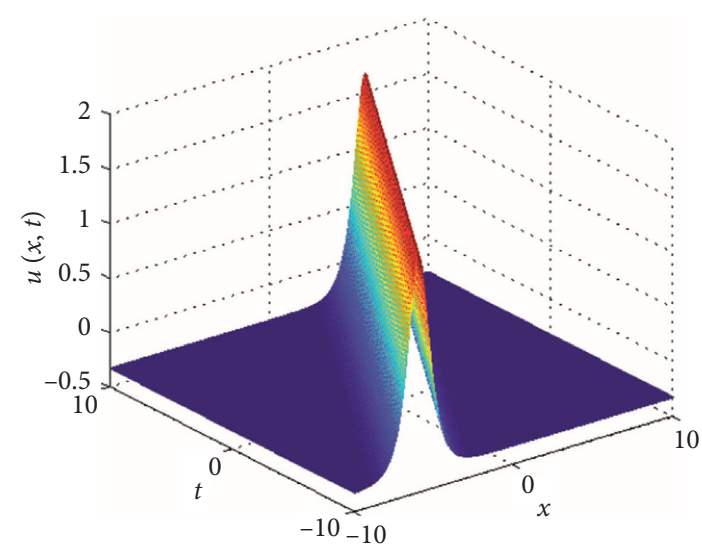

Figure 7: The $3 \mathrm{~d}$ surface of equation (73) for the values $\alpha=0.5, \beta=-1, \gamma=1$, and $B=2$.

$$
u(x, t)=-\frac{\alpha}{3 \beta}-B \sqrt{\frac{2 \gamma}{\beta}} \operatorname{sech}\left(B x-\frac{3 B^{3} \beta \gamma-B \alpha^{2}}{3 \beta} t\right),
$$

for a $W$-shaped soliton pulse, where $B$ is an optional constant.
The correctness of all given solutions has been confirmed with Maple by substituting them back into the original equation.

To better understand the characteristics of the soliton solution, we plot equations (57), (65), (73) (80), and (81) of equation (1) by taking different values of parameters $\alpha, \beta$, and $\gamma$ within the interval $(x, t) \in[-10,10] \times[-10,10]$ in Figures 1-7, respectively.

\section{Concluding Remarks and Observations}

In this research, we exerted the improved $\tan (\Theta(\vartheta))$-expansion and wave ansatz method as two useful mathematical tools to construct solitary solutions for the Gardner equation. These two methods for equation (1) have not been reported in the literature so far, to achieve the category of bright, dark, singular, and $W$-shaped soliton solutions. For a better understanding of the solutions, numerical results have also been included. On the other hand, the results are quite reliable for solving the Gardner equation. The results attest to the efficiency of the proposed method. These two powerful methods can also be applied to other nonlinear partial differential equations with time-dependent coefficients and their systems.

\section{Data Availability}

No data were used to support this study.

\section{Conflicts of Interest}

The authors declare that they have no conflicts of interest.

\section{References}

[1] C. S. Gardner, J. M. Greene, M. D. Kruskal, and R. M. Miura, "Method for solving the korteweg-deVries equation," Physical Review Letters, vol. 19, no. 19, pp. 1095-1097, 1967.

[2] R. M. Miura, C. S. Gardner, and M. D. Kruskal, "Korteweg-de vries equation and generalizations. II. existence of conservation laws and constants of motion," Journal of Mathematical Physics, vol. 9, no. 8, pp. 1204-1209, 1968.

[3] R. Grimshaw, D. Pelinovsky, E. Pelinovsky, and T. Talipova, "Wave group dynamics in weakly nonlinear long-wave models," Physica D: Nonlinear Phenomena, vol. 159, no. 1-2, pp. 35-57, 2001.

[4] S. Hamdi, B. Morse, B. Halphen, and W. Schiesser, "Analytical solutions of long nonlinear internal waves: part I," Natural Hazards, vol. 57, no. 3, pp. 597-607, 2011.

[5] K. Hosseini, M. S. Osman, M. Mirzazadeh, and F. Rabiei, "Investigation of different wave structures to the generalized third-order nonlinear scrödinger equation," Optik, vol. 206, Article ID 164259, 2020.

[6] K. Hosseini, M. Mirzazadeh, M. Ilie, and J. F. Gómez-Aguilar, "Biswas-arshed equation with the beta time derivative: optical solitons and other solutions," Optik, vol. 217, p. 164801, 2020.

[7] K. Hosseini, M. Mirzazadeh, J. Vahidi, and R. Asghari, "Optical wave structures to the fokas-lenells equation," Optik, vol. 207, Article ID 164450, 2020.

[8] M. M. A. Khater, "Novel exact solutions of the fractional bogoyavlensky-konopelchenko equation involvingthe 
atangana-baleanu-riemann derivative," Alexandria Engineering Journal, vol. 42, pp. 7210-7221, 2020.

[9] KK. Ali, H. Dutta H, R. Yilmazer et al., "On the new wave behaviors of the gilson-pickering equation," Frontiers in Physics, vol. 8, p. 54, 2020.

[10] H. M. Srivastava, H. Günerhan, and B. Ghanbari, "Exact traveling wave solutions for resonance nonlinear schrödinger equation with intermodal dispersions and the Kerr law nonlinearity," Mathematical Methods in the Applied Sciences, vol. 42, no. 18, pp. 7210-7221, 2019.

[11] D. Lu, M. Suleman, J. H. He, U. Farooq, S. Noeiaghdam, and F. A. Chandio, "Elzaki projected differential transform method for fractional order system of linear and nonlinear fractional partial differential equation," Fractals, vol. 26, no. 03, p. 1850041, 2018.

[12] B. Ghanbari and J. Liu, "Exact solitary wave solutions to the (2+1)dimensional generalised camassa-holm-kadomtsev-petviashvili equation,” Pramana - Journal of Physics, vol. 94, p. 21, 2020.

[13] J. Manafian, M. Lakestani, and A. Bekir, "A new analytical approach to solve some of the fractional-order partial differential equations," Indian Journal of Physics, vol. 91, no. 3, pp. 243-258, 2016.

[14] J. Manafian and M. Lakestani, "Optical soliton solutions for the Gerdjikov-Ivanov model via $\tan (\Theta / 2)$-expansion method," Optik, vol. 20, no. 127, pp. 9603-9620, 2016.

[15] J. Manafian, "Optical soliton solutions for Schrödinger type nonlinear evolution equations by the $\tan (\Theta / 2)$-expansion method," Optik, vol. 10, no. 127, pp. 4222-4245, 2016.

[16] J. Manafian and M. Lakestani, “Abundant soliton solutions for the Kundu-Eckhaus equation via $\tan (\Theta(\vartheta))$-expansion method," Optik, vol. 127, no. 14, pp. 5543-5551, 2016.

[17] K. Hosseini, M. Mirzazadeh, M. Ilie, and S. Radmehr, "Dynamics of optical solitons in the perturbed gerdjikov-ivanov equation," Optik, vol. 206, p. 164350, 2020.

[18] K. Hosseini, M. Mirzazadeh, F. Rabiei, H. M. Baskonus, and G. Yel, "Dark optical solitons to the biswas-arshed equation with high order dispersions and absence of the self-phase modulation," Optik, vol. 209, Article ID 164576, 2020.

[19] EM. E. Zayed and A. G. Al-Nowehy, "The solitary wave ansatz method for finding the exact bright and dark soliton solutions of two nonlinear schrödinger equations," Journal of the Association of Arab Universities for Basic and Applied Sciences, vol. 1, no. 24, pp. 184-190, 2017.

[20] I. Bendahmane, H. Triki, A. Biswas et al., "Bright, dark and W-shaped solitons with extended nonlinear schrödinger's equation for odd and even higher-order terms," Superlattices and Microstructures, vol. 114, pp. 53-61, 2018.

[21] S. Aouadi, A. Bouzida, A. K. Daoui et al., "W-shaped, bright and dark solitons of biswas-arshed equation," Optik, vol. 182, pp. 227-232, 2019.

[22] Z. Triki, S. Liu, and S. Liu, "New kinds of solutions to gardner equation," Chaos, Solitons \& Fractals, vol. 20, no. 2, pp. 301-309, 2004.

[23] G. Xu, Z. Li, and Y. Liu, "Exact solutions to a large class of nonlinear evolution equations," Chinese Journal of Physics, vol. 41, pp. 232-241, 2003.

[24] A.-M. Wazwaz, "New solitons and kink solutions for the gardner equation," Communications in Nonlinear Science and Numerical Simulation, vol. 12, no. 8, pp. 1395-1404, 2007.

[25] E. V. Krishnan, H. Triki, H. Labidi, and A. Biswas, "A study of shallow water waves with gardner's equation," Nonlinear Dynamics, vol. 66, no. 4, pp. 497-507, 2011.
[26] A. M. Wazwaz and M. Lakestani, "Solitons and singular solitons for the gardner-KP equation," Applied Mathematics and Computation, vol. 204, pp. 162-169, 2008.

[27] M. Kumar and D. V. Tanwar, "On lie symmetries and invariant solutions of $(2+1)$-dimensional gardner equation," Communications in Nonlinear Science and Numerical Simulation, vol. 69, pp. 45-57, 2019.

[28] B. Ghanbari and D. Baleanu, "New solutions of gardner's equation using two analytical methods," Frontiers in Physics, vol. 7, p. 202, 2019.

[29] Z. Korpinar, M. Inc, D. Baleanu et al., "Theory and application for the time fractional Gardner equation with Mittag-Leffler kernel," Journal of Taibah University for Science, vol. 1, no. 13, pp. 813-819, 2019.

[30] J. Zhang, "Exact and explicit solitary wave solutions to some nonlinear equations," International Journal of Theoretical Physics, vol. 35, no. 8, pp. 1793-1798, 1996.

[31] M. Wadati, "Wave propagation in nonlinear lattice. II," Journal of the Physical Society of Japan, vol. 38, no. 3, pp. 681-686, 1975.

[32] M. Wadati, "Wave propagation in nonlinear lattice. I," Journal of the Physical Society of Japan, vol. 38, no. 3, pp. 673-680, 1975.

[33] Y. kai Liu and B. Li, "Nonlocal symmetry and exact solutions of the (2+1)-dimensional Gardner equation," Chinese Journal of Physics, vol. 54, no. 5, pp. 718-723, 2016.

[34] G. Betchewe, K. K. Victor, B. B. Thomas, and K. T. Crepin, "New solutions of the Gardner equation: analytical and numerical analysis of its dynamical understanding," Applied Mathematics and Computation, vol. 223, pp. 377-388, 2013.

[35] G. Yildiz and Ç. Turkmen, "Application of kudryashov method to some equations used in physics science," Journal of Science and Technology, vol. 3, no. 12, pp. 1485-1492, 2019.

[36] J. Fei, W. Cao, and Z. Ma, "Nonlocal symmetries and explicit solutions for the Gardner equation," Applied Mathematics and Computation, vol. 314, pp. 293-298, 2017.

[37] M. A. Alejo, "On the ill-posedness of the Gardner equation," Journal of Mathematical Analysis and Applications, vol. 396, no. 1, pp. 256-260, 2012.

[38] D. Cao, "The classification of the single traveling wave solutions to the time-fraction Gardner equation," Chinese Journal of Physics, vol. 59, pp. 79-92, 2019.

[39] A.-M. Wazwaz, "A study on KdV and Gardner equations with time-dependent coefficients and forcing terms," Applied Mathematics and Computation, vol. 217, no. 5, pp. 2277-2281, 2010.

[40] S. Kumar, K. Singh, and R. K. Gupta, "Dynamics of internal waves in a stratified ocean modeled by the extended Gardner equation with time-dependent coefficients," Ocean Engineering, vol. 70, pp. 81-87, 2013.

[41] H. Triki, A. Biswas, S. P. Moshokoa, and M. Belic, "Optical solitons and conservation laws with quadratic-cubic nonlinearity," Optik, vol. 128, pp. 63-70, 2017. 